\title{
Conquering the White Cataract
}

\author{
Kate Xie and Marjan Farid \\ University of California-IIvine, Gavin Herbert Eye Institute, Irvine, CA, US
}

DOl: https://doi.org/10.17925/USOR.2018.11.1.24

W e review clinical pearls in the evaluation and surgical management of white cataracts. Femtosecond laser-assisted cataract surgery is a newer technology that can aid in capsulorhexis creation, however the surgeon should be aware of the risk of incomplete capsulotomy creation in intumescent white cataracts. The surgeon should be fully prepared and anticipate challenges at every step of cataract surgery in these complex cases.

\section{Keywords}

Dense cataract, femtosecond laser-assisted cataract surgery, intumescent cataract

Disclosure: Kate Xie and Marjan Farid have nothing to disclose in relation to this article.

Review Process: This article is a short opinion piece and was reviewed by the editorial board before publication.

Authorship: All named authors meet the International Committee of Medical Journal Editors (ICMJE) criteria for authorship of this manuscript, take responsibility for the integrity of the work as a whole, and have given final approval to the version to be published.

open Access: This article is published under the creative Commons Attribution Noncommercial License, which permits any noncommercial use, distribution, adaptation, and reproduction provided the original author(s) and source are given appropriate credit. (C) The Authors 2018.

Received: February 3, 2018

Published Online: March 5, 2018

Citation: US Ophthalmic Review, 2018;11(1):24-25

Corresponding Author: Marjan Farid, MD, University of California-Irvine, Gavin Herbert Eye Institute, 850 Health Sciences Road, Irvine, CA 9269, US. E: mfarid@uci.edu

Support: No funding was received in

the publication of this article.
The key to a successful white cataract surgery is to be prepared.

This starts with a thorough preoperative evaluation. A careful slit lamp exam is important-if the anterior lens is bulging, this could be a sign of increased posterior intralenticular pressure. Does the cortex look formed or milky? Can you see a brunescent nuclear center? Is there scarring of the anterior capsule? Inquire about a history of trauma and look carefully for areas of potential zonular weakness. Brazitikos et al described a classification system of white cataracts; those with liquefied cortex were at most risk for rapid radialization of the capsular tear, known as the "Argentinian flag sign." White cataracts with a solid cortex and nucleus were more dense and sometimes adherent to the posterior lens capsule, presenting a challenge during phacoemulsification. ${ }^{1}$

Arguably the most difficult aspect of a white cataract surgery is the creation of the capsulorhexis. If the red reflex is compromised, there can be high intracapsular tension due to accumulation of liquefied lens protein, and there can be anterior capsular scarring. Not only should the surgeon be prepared to use trypan blue stain to help with visualization for the capsulorhexis, but should also consider using a heavy molecular weight viscoelastic agent to flatten the capsule and equalize the pressure gradient between the lens and anterior chamber. In these cases, it is always better to begin with a smaller capsulorhexis with subsequent enlargement, which can be done even after the intraocular lens has been placed and centered within the capsular bag. Aspiration with a 27- or 30-gauge needle prior to initiation of the capsulorhexis may be beneficial to decompress a turgid lens and prevent radialization of the capsulorhexis.

The development of femtosecond laser-assisted cataract surgery (FLACS) adds one more tool to our armament for approaching the white cataract, and may be especially useful in the creation of the capsulotomy. FLACS provides the opportunity to create a perfectly circular rhexis in one attempt. Instantaneous creation of the entire capsulorhexis avoids placing stress at the center of the lens, where intralenticular pressure is the highest. This theoretically reduces the chance of the dreaded "Argentinian flag sign." However, for very intumescent lenses, FLACS can cause a sudden release of milky fluid during the capsulotomy, obscuring the view and altering the lens position., ${ }^{2,3}$ This can result in an incomplete capsulotomy creation. The presence of anterior capsular scarring can also result in residual adhesions. A recent study by Titiyal et al. showed that in a group of patients who underwent FLACS for white cataract, roughly half of the capsulotomies were complete in cases where there was egress of milky fluid-the other half were incomplete capsulotomies where there were either residual microadhesions or there were 1-2 clock hours of incomplete area of capsulotomy. ${ }^{3}$ 
After the capsulorhexis has been successfully created, do not let your guard down. Either a milky soft cataract or a very dense and hard endonucleus will be left. Hydrodissection can inadvertently lead to trapping of fluid posterior to a dense nucleus, increasing posterior pressure and resulting in blowout of the posterior capsule. Gentle hydrodissection and mobilizing the nucleus to release any posterior pressure is key. When it comes to nuclear disassembly, the use of phaco quick-chop to keep the instruments centered and minimize phaco energy can be very useful in the case of a very dense nucleus. Adjusting the phaco sleeve so that more of the tip is exposed can help to obtain a better purchase of the lens. Be mindful that there may be very little epinuclear shell protecting the posterior capsule, as the cortex may have fully liquefied or the nucleus may have achieved an extreme density. In these cases, a thin layer of a dispersive viscoelastic placed behind the lens to create a "visco vault" or artificial epinucleus can aid in protecting the posterior capsule from trauma.

In summary, the surgeon should be prepared with the proper instrumentation, necessary tools, and surgical techniques to create a functional capsulotomy, capsular centration, and safe evacuation of the nucleus when operating on a white cataract. The way to approach these cases is with full preparation and a cool head. $\square$

1. Brazitikos PD, Tsinopoulos IT, Papadopoulos NT, et al., Ultrasonographic classification and phacoemulsification of white senile cataracts. Ophthalmology. 1999;106:2178-83.

2. Conrad-Hengerer I, Hengerer FH, Joachim SC, et al., Femtosecond laser-assisted cataract surgery in intumescent white cataracts. J Cataract Refract Surg. 2014;40:44-50.

3. Titiyal JS, Kaur M, Singh A, et al., Comparative evaluation of femtosecond laser-assisted cataract surgery and conventional phacoemulsification in white cataract. Clin Ophthalmol. 2016;10:1357-64. 